Sharif University of Technology
Scientia Iranica
Transactions E: Industrial Engineering
http://scientiairanica.sharif.edu
IRAN ICA

\title{
An application of genetic algorithm and PSO in an inventory model for a single deteriorating item with variable demand dependent on marketing strategy and displayed stock level
}

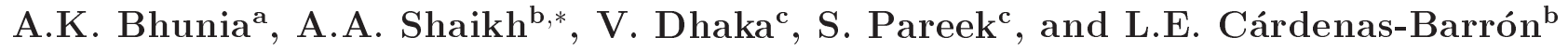 \\ a. Department of Mathematics, The University of Burdwan, Burdwan-713104, India. \\ b. School of Engineering and Sciences, Tecnológico de Monterrey, Ave. E. Garza Sada 2501 Sur, C.P. 64849, Monterrey, Nuevo \\ León, México. \\ c. Department of Mathematics \& Statistics, Banasthali University, Banathali, India.
}

Received 22 April 2015; received in revised form 14 October 2016; accepted 6 March 2017

\author{
KEYWORDS \\ Inventory; \\ Deteriorating; \\ Variable demand; \\ Display stock level; \\ Genetic algorithm; \\ Particle swarm \\ optimization.
}

\begin{abstract}
This paper deals with an inventory model applied to a single deteriorating item considering the impact of marketing decisions and displaced stock level on the demand. Partial backlogged shortages are allowed. Different scenarios have been investigated through analyzing the shop storage capacity and demand parameters. For each scenario, the corresponding problem has been formulated as a nonlinear mixed integer optimization problem and solved by a real coded genetic algorithm and a particle swarm optimization technique. To illustrate the inventory model, a numerical example has been solved and sensitivity analyses have been done numerically to study the effect of changes of different parameters on the optimal policies.
\end{abstract}

(C) 2018 Sharif University of Technology. All rights reserved.

\section{Introduction}

In the existing literature of inventory control theory, it is observed that the conventional inventory models have been developed under the assumption that the lifetime of an item is infinite during the stock-in period, implying that the utility of an item does not change in the stock-in period and is fully usable for satisfying any future demand. However, this assumption is not always true for some commonly used physical products, such as wheat, paddy or any other type of food grains,

\footnotetext{
*. Corresponding author.

E-mail addresses: akbhunia@math.buruniv.ac.in (A.K. Bhunia); aakbarshaikh@itesm.mx (A.A. Shaikh);

dhakavinti@gmail.com (V. Dhaka); psarla13@gmail.com (S. Pareek); lecarden@tec.mx (L.E. Cárdenas-Barrón)
}

vegetables, fruits, among others, due to the deterioration effect. A certain fraction of these products is either damaged or decayed, or affected by some other factors. In some cases, the deteriorated units are not completely lost due to good inventory management. These units can be sold with a reduced price, obviously less than the purchase cost. Hence, the deterioration effect plays a crucial role in the inventory control theory. For highly perishable goods, the loss due to the deterioration effect can either be avoided or reduced by fulfilling the backlogged demand, and the procurement of products can be done economically. Hence, the problem of finding a proper trade-off between these items is a highly complex problem, and this type of problem has naturally received much attention in the inventory literature. In this area, a large number of mathematical models have been reported in the existing literature. Among those, to get an idea of the 
trends of the recent research, one may refer to the works of Mondal and Phaujdar [1], Pakkla and Achary [2], Goyal and Gunasekaran [3], Giri et al. [4], Bhunia and Maiti [5,6], Luo [7], Chang and Dye [8], Abad [9], Pal et al. [10], Dye et al. [11], Pal et al. [12], Teleizadeh et al. [13], Bhunia et al. [14], Bhunia and Shaikh [15,16], Liang and Zhou [17], Yang [18], Teleidadeh et al. [19], Teleidadeh et al. [20], Bhunia et al. [21], Yang and Chang [22], Teleizadeh et al. [23,24], Teleizadeh et al. [25], Bhunia and Shaikh [26], Bhunia et al. [27], Bhunia et al. [28], Bhunia et al. [29], and others.

In the current competitive market situation, a product is promoted in the society through the glamorous advertisement in the electronic, print media and/or by an attractive display of units in the showroom at the market. Glamorous display of the large number of units with modern light and electronic arrangements attracts people to purchase more. Observing this phenomenon, most of the marketing researchers and practitioners have become motivated to investigate its modeling aspects. Basically, they concluded that the demand rate of an item is dependent on the displayed stock-level in the showroom/shop. Again, time-to-time advertisement (through the wellknown media such as TV, Radio, Newspaper, Magazine, internet, etc. and also through the sales representatives) of an item and its selling price variations also change the demand of that item. Therefore, it can be concluded that there exists a functional relationship among the demand of an item, displayed stock-level in a showroom/shop, selling price, and the frequency/cost of the advertisement of that item. To the best of our knowledge, this type of demand was first reported in the inventory models developed by $\mathrm{Pal}$ et al. [10] and Mondal et al. [30]. On the one hand, considering only the displayed stock-level dependent demand, Baker and Urban [31], Mondal and Phaujdar [1], Datta and Pal [32], Urban [33], Paul et al. [34], Giri et al. [4], and others developed different types of inventory models. On the other hand, very few researchers studied the effects of price variations and/or the advertisement on the demand of an item. Among them, one may refer to the works of Urban [35], Goyal and Gunasekaran [3], Bhunia and Maiti [5], Luo [7], Abad [9,36], Pal et al. [10,37], Bhunia and Shaikh [15], Bhunia and Shaikh [26] and Bhunia et al. [29], Sarkar [38-40], Sarkar et al. [41,42], Sarkar and Sarkar [43,44], among others.

Generally, the decision-making problems are solved by traditional direct or gradient-based optimization methods. There are some shortcomings in the use of these methods. One such shortcoming is that the traditional non-linear optimization methods are very often stuck to the local optima. To avoid some of these shortcomings, one of the soft computing methods, Genetic Algorithm (GA), is used for solving decision- making problems. It is a computerized stochastic search and optimization algorithm based on the mechanics of natural selection and natural genetics [45]. It is executed iteratively on the set of either real or binary coded solutions called population. In each iteration (which is called generation), three basic genetic operations, i.e. selection/reproduction, crossover, and mutation, are performed. Prof. J.H. Holland of University of Michigan envisaged the fundamental concept of this algorithm in the mid-seventies and published his seminal work [46]. Thereafter, a number of researchers have contributed to the development of this field. Detailed discussions on the development of this subject are available in the books of Goldberg [45], Michalawicz [47], Deb [48], Sakawa [49], and others.

Over the last decade, this algorithm has been successfully applied to solving different types of decisionmaking problems such as travelling salesman problems, scheduling problems, numerical optimization, etc. However, till now, only a very few researchers have applied it to solve problems in the field of inventory control system.

This paper develops an inventory model for a single deteriorating item considering the impact of marketing strategies, such as pricing of the item and advertising as well as the displayed stock level, on the demand rate of that item. The storage capacity of the showroom/shop is assumed to be finite. Shortages, if any, are allowed and partially backlogged with a rate dependent on the duration of the waiting time up to the next replenishment. By analyzing the storage capacity of the showroom/shop and displayed stock level dependency parameters of demand rate, different scenarios have been investigated. In each scenario, the corresponding problem has been formulated as a non-linear mixed integer optimization problem with three decision variables: one as integer and other two as non-integer (continuous) variables. Considering the complexity of solving such problems for different scenarios, a Real-Coded Genetic Algorithm (RCGA) is developed for mixed variables (integer and continuous) with ranking selection, whole arithmetical crossover, non-uniform mutation for non-integer variables, and uniform mutation for the integer variable. In order to illustrate the results of different scenarios, a numerical example has been considered and solved. Finally, sensitivity analyses have been performed numerically to study the variations of different system parameters on the initial on-hand inventory level, shortage level, and cycle length along with the maximum total profit per time unit.

\section{Notation and assumptions}

The following assumptions and notation are used in developing the proposed inventory model. 


\subsection{Notation}

Parameters:

$C_{1} \quad$ Inventory carrying cost per unit item per unit of time $(\$ /$ unit/time unit)

$C_{2} \quad$ Shortage cost per unit item per unit of time (\$/unit/time unit)

$C_{3} \quad$ Purchase cost per unit item (\$/unit)

$C_{4} \quad$ Ordering cost per cycle $(\$ /$ cycle $)$

$p \quad$ Selling price per unit item (\$/unit)

$p^{\prime}\left(\leq C_{3}\right) \quad$ Salvage value associated to the deteriorating unit during the cycle per unit item (\$/unit)

$\lambda, m \quad$ Markup rate $(\lambda>1,0<m<1)$, i.e. $p=\lambda C_{3}, p^{\prime}=m C_{3}$

$G \quad$ Advertising cost per advertisement (\$/advertisement)

$W \quad$ Storage capacity of the showroom/shop (units)

$q \quad$ Order quantity of the cycle (units)

$q(t) \quad$ Instantaneous displayed stock level at time $t$ (units)

$D(A, p, q) \quad$ Demand rate (units/time unit), which is dependent on frequency of advertisement $(A)$, unit selling price and instantaneous displayed stock level

$S_{0}, S_{1} \quad$ Displayed stock level dependency demand parameters

$\theta \quad$ Constant deterioration rate

$\delta \geq 0 \quad$ Backlogging parameter

$D^{\prime} \quad$ Total deteriorated units over the stock-in period (units)

$C_{\text {sho }} \quad$ Total shortage cost over the entire cycle $(\$)$

TC Total cost $(\$)$

$X \quad$ Net profit $(\$)$

$Z_{j} \quad$ Total profit or profit function of scenario $j$ ( $\$ /$ time unit)

Dependent variables:

$t_{0} \quad$ Time period when displayed stock is $S_{1}$ (time unit)

$t_{1} \quad$ Time period when displayed stock is $S_{0}$ (time unit)

$t_{2} \quad$ Time period when inventory level is zero (time unit)

$t_{3} \quad$ Time period when inventory level reaches maximum shortage level (time unit)

$T \quad$ Total cycle length (time unit)
Decision variables:

$A \quad$ Frequency of the advertisement in the cycle (an integer number)

$S(\leq W) \quad$ Initial stock level (units)

$R \quad$ The highest shortage quantity due to partial backlogging (units)

For PSO

p_size Population size

$m_{-}$gen Maximum generation

$c_{1}(>0) \quad$ Cognitive learning rate

$c_{2}(>0) \quad$ Social learning rate

$r_{1}, r_{2} \quad$ Random number which is uniformly lying between $[0,1]$

$x_{i}^{(k)} \quad$ Velocity of the $i$ th particle at the $k$ th generation/iteration

$p_{i}^{(k)} \quad$ Best previous position of the $i$ th particle at the $k$ th iteration

$p_{g}^{(k)} \quad$ Position of the best particle among all other particles in the population

$\chi \quad$ Constriction factor

\subsection{Assumptions}

(i) Replenishment rate is infinite and lead time is constant;

(ii) The entire lot is delivered in one batch;

(iii) The inventory planning horizon is infinite and the inventory system involves only one item and one stocking point;

(iv) The deterioration rate is constant;

(v) The deteriorated units are not replaced, but those units are sold with a reduced price;

(vi) The storage capacity of a showroom/shop is limited;

(vii) Demand rate, $D(A, p, q)$, is a deterministic function of the selling price $(p)$ per unit, frequency of advertisement $(A)$, and the displayed inventory level in the show-room within displayed levels $S_{0}$ to $S_{1}$; beyond this range, it becomes constant with respect to the displayed inventory level. It takes the following forms:

$$
\begin{aligned}
D(A, p, q) & =f\left(A, p, S_{1}\right) \quad \text { for } \quad q>S_{1}, \\
& =f(A, p, q) \quad \text { for } \quad S_{0}<q \leq S_{1}, \\
& =f\left(A, p, S_{0}\right) \quad \text { for } \quad q \leq S_{0},
\end{aligned}
$$

where $f(A, p, q)=A^{\gamma}(a-b p+c q), a, b, c, \gamma \geq 0$.

It is to be noted that $f(A, p, q)$ is a differentiable function of $q$ for the fixed values of $A$ and $p$. 
(viii) Shortages, if any, are allowed and partially backlogged. During the stock-out period, the backlogging rate is dependent on the length of the waiting time up to the arrival of fresh lot. Considering this situation, the rate is defined as $[1+\delta(T-t)]^{-1}$.

\section{Inventory model description and analysis}

It is assumed that, after fulfilling the backorder quantity, the on-hand inventory level is $S$ at $t=0$ and declines continuously due to the deterioration effect of the item and also to meet up the customer's demand. This process is continued up to the time when the on-hand inventory level reaches zero. Then, shortage occurs and accumulates at the rate $[1+\delta(T-t)]^{-1}$, $(\delta \geq 0)$ up to time $t=T$ when a next lot arrives. At time $t=T$, the maximum shortage level is $R$. This entire cycle then repeats itself after cycle length, $T$. The problem is to determine the optimal values of $S$ and $R$, such that the total profit per time unit of the system is maximized and also the corresponding value of $T$ is determined.

By analyzing the model and relative size of the displayed stock level dependency parameters $S_{0}$, $S_{1}$, and the finite storage capacity $W$ of the showroom/shop, the following scenarios may arise:

Scenario 1: $S_{1}<S \leq W$,

Scenario 2: $S_{0}<S \leq W \leq S_{1}$,

Scenario 3: $S<W \leq S_{0}$.

Now, all the scenarios are discussed in detail one after another below.

Scenario 1: $S_{1}<S \leq W$

In this scenario, the order quantity is $Q=S+R$. Following the earlier mentioned assumptions, the differential equations representing the displayed inventory level, $q(t)$, at any instant $t$ are given by:

$$
\begin{aligned}
& \frac{d q(t)}{d t}+\theta q(t)=-f\left(A, p, S_{1}\right), \quad 0 \leq t<t_{0} \\
& \frac{d q(t)}{d t}+\theta q(t)=-f(A, p, q), \quad t_{0} \leq t<t_{0}+t_{1} \\
& \frac{d q(t)}{d t}+\theta q(t)=-f\left(A, p, S_{0}\right) \\
& t_{0}+t_{1} \leq t<t_{0}+t_{1}+t_{2} \\
& \frac{d q(t)}{d t}=-f\left(A, p, S_{0}\right) /[1+\delta(T-t)] \\
& t_{0}+t_{1}+t_{2} \leq t \leq t_{0}+t_{1}+t_{2}+t_{3}(=T)
\end{aligned}
$$

subject to the conditions that:

$$
\begin{aligned}
& q(t)=S \quad \text { at } \quad t=0, \\
& q(t)=S_{1} \quad \text { at } \quad t=t_{0}, \\
& q(t)=S_{0} \quad \text { at } \quad t=t_{0}+t_{1}, \\
& q(t)=0 \quad \text { at } \quad t=t_{0}+t_{1}+t_{2}, \\
& q(t)=-R \quad \text { at } \quad t=T .
\end{aligned}
$$

It is also assumed that instantaneous stock level, $q(t)$, is continuous at $t=t_{0}, t=t_{0}+t_{1}$, and $t=t_{0}+t_{1}+$ $t_{2}$. The inventory level through time for Scenario 1 is represented in Figure 1.

Using Conditions (2a)-(2d), the solutions to differential Eqs. (1a)-(1d) are as follows:

$$
\begin{aligned}
q(t)= & {\left[S+f\left(A, p, S_{1}\right) / \theta\right] \exp (-\theta t)-f\left(A, p, S_{1}\right) / \theta } \\
0 \leq & t<t_{0} \\
q(t)= & \left\{S_{1}+A^{\gamma}(a-b p) /\left(A^{\gamma} c+\theta\right)\right\} \\
& \exp \left\{\left(A^{\gamma} c+\theta\right)\left(t_{0}-t\right)\right\}-A^{\gamma}(a-b p) /\left(A^{\gamma} c+\theta\right) \\
t_{0} \leq & t<t_{0}+t_{1}, \\
q(t)= & \left\{S_{0}+f\left(A, p, S_{0}\right) / \theta\right\} \\
& \exp \left\{\theta\left(t_{0}+t_{1}-t\right)\right\} f\left(A, p, S_{0}\right) / \theta \\
t_{0}+ & t_{1} \leq t<t_{0}+t_{1}+t_{2} \\
q(t)= & f\left(A, p, S_{0}\right) \log |1+\delta(T-t)| / \delta-R \\
t_{0}+ & t_{1}+t_{2} \leq t<t_{0}+t_{1}+t_{2}+t_{3}(=T)
\end{aligned}
$$

Using the continuity of $q(t)$ at $t=t_{0}$, it follows that:

$$
S_{1}=\left[S+f\left(A, p, S_{1}\right) / \theta\right] \exp \left(-\theta t_{0}\right)-f\left(A, p, S_{1}\right) / \theta,
$$

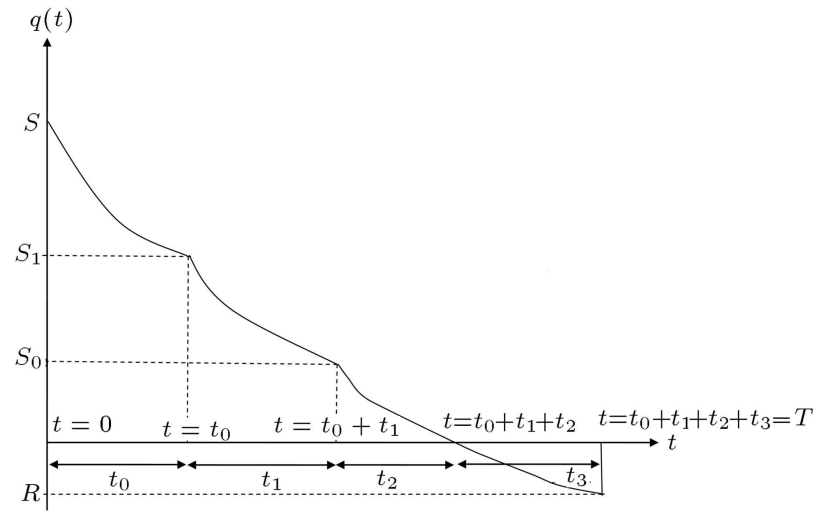

Figure 1. Inventory behavior of Scenario 1. 
implying that:

$$
t_{0}=\frac{1}{\theta} \log \left|\frac{f\left(A, p, S_{1}\right)+\theta S}{f\left(A, p, S_{1}\right)+\theta S_{1}}\right| .
$$

Again, using the continuity condition of $q(t)$ at $t=$ $t_{0}+t_{1}$, hence:

$$
\begin{aligned}
S_{0}= & \left\{S_{1}+A^{\gamma}(a-b p) /\left(A^{\gamma} c+\theta\right)\right\} \\
& \exp \left\{-\left(A^{\gamma} c+\theta\right) t_{1}\right\}-A^{\gamma}(a-b p) /\left(A^{\gamma} c+\theta\right),
\end{aligned}
$$

which implies that:

$$
t_{1}=\frac{1}{A^{\gamma} c+\theta} \log \left|\frac{\left(A^{\gamma} c+\theta\right) S_{1}+A^{\gamma}(a-b p)}{\left(A^{\gamma} c+\theta\right) S_{0}+A^{\gamma}(a-b p)}\right| .
$$

As $q(t)$ is continuous at $t=t_{0}+t_{1}+t_{2}$ and then from Eqs. (3c) and (3d):

$$
t_{2}=\frac{1}{\theta} \log \left|\frac{f\left(A, p, S_{0}\right)+\theta S_{0}}{f\left(A, p, S_{0}\right)}\right| .
$$

Again, from $q(t)=0$ at $t=t_{0}+t_{1}+t_{2}$ and Eq. (3d):

$$
t_{3}=\left[\exp \left(\frac{\delta R}{f\left(A, p, S_{0}\right)}\right)-1\right] / \delta \text {. }
$$

During the stock-in period, the deteriorated units have to be separated. The total deteriorated units over the stock-in period $\left(0 \leq t \leq t_{0}+t_{1}+t_{2}\right)$ are given by:

$$
D^{\prime}=\theta \int_{0}^{t_{0}+t_{1}+t_{2}} q(t) d t
$$

then:

$$
\int_{0}^{t_{0}+t_{1}+t_{2}} q(t) d t=\frac{D^{\prime}}{\theta}
$$

This means that the inventory carrying units over the interval $0 \leq t \leq t_{0}+t_{1}+t_{2}$ are $\frac{D^{\prime}}{\theta}$.

Again, the difference between the initial and total selling units during the stock-in period of the entire cycle will be also deteriorated units as follows:

$$
\begin{aligned}
D^{\prime}= & S-\int_{0}^{t_{0}+t_{1}+t_{2}} D(A, p, q) d t \\
D^{\prime}= & S-f\left(A, p, S_{1}\right) t_{0}-f\left(A, p, S_{0}\right) t_{2} \\
& -A^{\gamma}(a-b p) t_{1}-A^{\gamma} c \int_{t_{0}}^{t_{0}+t_{1}} q(t) d t .
\end{aligned}
$$

The total shortage cost over the entire cycle is given by:

$$
\begin{aligned}
C_{\text {sho }} & =C_{2} \int_{t_{0}+t_{1}+t_{2}}^{T}[-q(t)] d t \\
& =C_{2} f\left(A, p, S_{0}\right)\left[t_{3}-\log \left|1+\delta t_{3}\right| / \delta\right] / \delta .
\end{aligned}
$$

During the entire inventory cycle, the Total Cost $(T C)$ of the scenario is given by:

$$
\begin{aligned}
T C= & <\text { ordering cost }>+<\text { purchasing cost }> \\
& +<\text { inventory carrying cost }> \\
& +<\text { inventory shortage cost }> \\
& +<\text { advertisement cost }>
\end{aligned}
$$

$$
T C=C_{4}+C_{3}(S+R)+C_{1} \frac{D^{\prime}}{\theta}+C_{\text {sho }}+A G .
$$

Therefore, the net profit $(X)$ for this scenario during the cycle of time-span is the difference between the sales revenue and the total cost of the system:

$$
\begin{aligned}
X(A, S, R)= & \left(p-C_{3}\right)(S+R)-\left(\frac{C_{1}}{\theta}+p-p^{\prime}\right) D^{\prime} \\
& -C_{\text {sho }}-A G-C_{4},
\end{aligned}
$$

where $S$ and $R$ are continuous variables, and $A$ is an integer variable.

Hence, for the fixed values of markup rates, $\lambda$ and $m$, profit function, $Z_{1}$, (total profit per unit time for the entire cycle) of Scenario 1 of the system is given by:

$$
Z_{1}(A, S, R)=\frac{X}{T}
$$

Hence, the nonlinear mixed integer optimization problem is:

maximizing $Z_{1}(A, S, R)$,

subject to $S_{1}<S \leq W, R>0$, and $A$ is an integer.

Scenario 2: $\quad S_{0}<S \leq W \leq S_{1}$

In this scenario, the order quantity is $Q=S+R$. The differential equations representing displayed inventory level, $q(t)$, at any instant $t$ are given by:

$$
\begin{aligned}
& \frac{d q(t)}{d t}+\theta q(t)=-f(A, p, q), \quad 0 \leq t<t_{1}, \\
& \frac{d q(t)}{d t}+\theta q(t)=-f\left(A, p, S_{0}\right), \quad t_{1} \leq t \leq t_{1}+t_{2} \\
& \frac{d q(t)}{d t}=-f\left(A, p, S_{0}\right) /[1+\delta(T-t)] \\
& t_{1}+t_{2}<t \leq t_{1}+t_{2}+t_{3}(=T)
\end{aligned}
$$




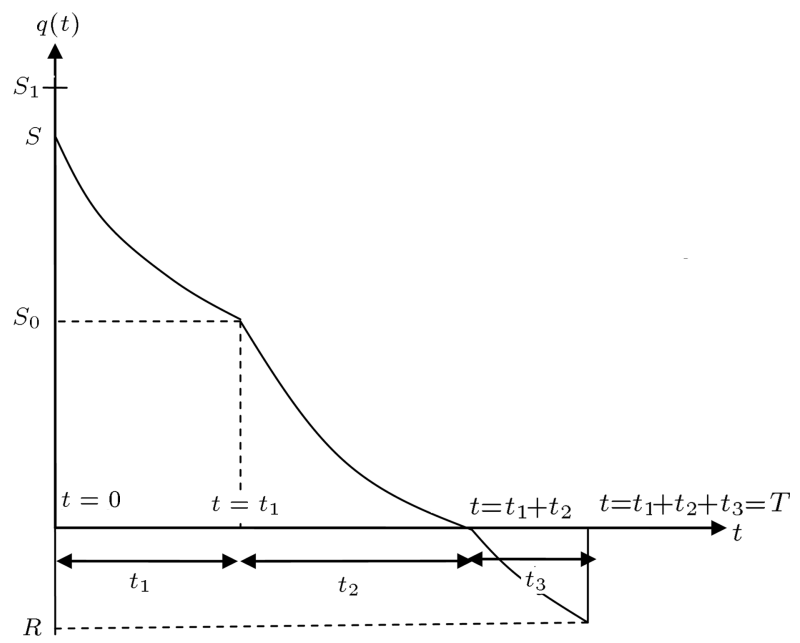

Figure 2. Inventory behavior of Scenario 2.

subject to the conditions that:

$$
\begin{aligned}
& q(t)=S \quad \text { at } \quad t=0, \\
& q(t)=S_{0} \quad \text { at } \quad t=t_{1}, \\
& q(t)=0 \quad \text { at } \quad t=t_{1}+t_{2}, \\
& q(t)=-R \quad \text { at } \quad t=T .
\end{aligned}
$$

Also, instantaneous stock level, $q(t)$, is continuous at $t=t_{1}$ and $t=t_{1}+t_{2}$. The inventory behavior through time for Scenario 2 is depicted in Figure 2.

Using Conditions (11a)-(11d), the solutions of differential Eqs. (10a)-(10c) are given by:

$$
\begin{aligned}
q(t)= & {\left[S+A^{\gamma}(a-b p) /\left(A^{\gamma} c+\theta\right)\right] } \\
& \exp \left(-t\left(A^{\gamma} c+\theta\right)\right)-A^{\gamma}(a-b p) /\left(A^{\gamma} c+\theta\right) \\
0 \leq & t<t_{1} \\
q(t)= & {\left[S_{0}+f\left(A, p, S_{0}\right) / \theta\right] } \\
& \exp \left(\theta\left(t_{1}-t\right)\right)-f\left(A, p, S_{0}\right) / \theta \\
t_{1} \leq & t \leq t_{1}+t_{2}, \\
q(t)= & f\left(A, p, S_{0}\right) \log |1+\delta(T-t)| / \delta-R \\
t_{1}+ & t_{2}<t \leq t_{1}+t_{2}+t_{3}(=T) .
\end{aligned}
$$

Now, from the continuity condition of $q(t)$ at $t=t_{1}$, it follows that:

$$
\begin{aligned}
S_{0}= & \left\{S+A^{\gamma}(a-b p) /\left(A^{\gamma} c+\theta\right)\right\} \exp \left\{-\left(A^{\gamma} c+\theta\right) t\right\} \\
& -A^{\gamma}(a-b p) /\left(A^{\gamma} c+\theta\right),
\end{aligned}
$$

which implies that:

$$
t_{1}=\frac{1}{A^{\gamma} c+\theta} \log \left|\frac{\left(A^{\gamma} c+\theta\right) S+A^{\gamma}(a-b p)}{\left(A^{\gamma} c+\theta\right) S_{0}+A^{\gamma}(a-b p)}\right| .
$$

Again, from $q(t)=0$ at $t=t_{1}+t_{2}$ and Eq. (12b):

$$
\left.\left[S_{0}+f\left(A, p, S_{0}\right) / \theta\right] \exp \left(-\theta t_{2}\right)\right]=f\left(A, p, S_{0}\right) / \theta,
$$

which implies that:

$$
t_{2}=\frac{1}{\theta} \log \left|\frac{f\left(A, p, S_{0}\right)+\theta S_{0}}{f\left(A, p, S_{0}\right)}\right| .
$$

As $q(t)$ is continuous at $t=t_{1}+t_{2}$, and hence from Eqs. (12b) and (12c),

$$
\begin{aligned}
& f\left(A, p, S_{0}\right) \log \left|1+\delta t_{3}\right|=\delta R, \\
& t_{3}=\frac{1}{\delta}\left[\exp \left\{\frac{\delta R}{f\left(A, p, S_{0}\right)}\right\}-1\right],
\end{aligned}
$$

which is same as Eq. (7).

In this scenario, the total deteriorated units over the stock in period $\left(0 \leq t \leq t_{0}+t_{1}+t_{2}\right)$ are given by:

$$
\begin{aligned}
& D^{\prime}=S-\int_{0}^{t_{1}+t_{2}} D(A, p, q) d t, \\
& D^{\prime}=S-f\left(A, p, S_{0}\right) t_{2}-A^{\gamma}(a-b p) t_{1}-A^{\gamma} c \int_{0}^{t_{1}} q(t) d t .
\end{aligned}
$$

The expression for $C_{\text {sho }}$ is the same as in Scenario 1.

Hence, the net profit for this scenario during time period $T$ is given by:

$$
\begin{aligned}
X(A, S, R)= & \left(p-C_{3}\right)(S+R)-\left(\frac{C_{1}}{\theta}+p-p^{\prime}\right) D^{\prime} \\
& -C_{\text {sho }}-A G-C_{4}
\end{aligned}
$$

where $S$ and $R$ are continuous variables and $A$ is an integer variable.

Therefore, for the fixed values of markup rates, $\lambda$ and $m$, profit function, $Z_{2}$, (total profit per time unit for the entire cycle) of Scenario 2 of the system is expressed as follows:

$$
Z_{2}(A, S, R)=\frac{X}{T}
$$

Thus, in this scenario, the optimization problem is:

maximizing $Z_{2}(A, S, R)$,

subject to $S_{0}<S \leq W \leq S_{1}, R>0$,

and $A$ is an integer.

Scenario 3: $\quad S \leq W \leq S_{0}$

The differential equations representing displayed inventory level, $q(t)$, at any instant, $t$, are given by: 


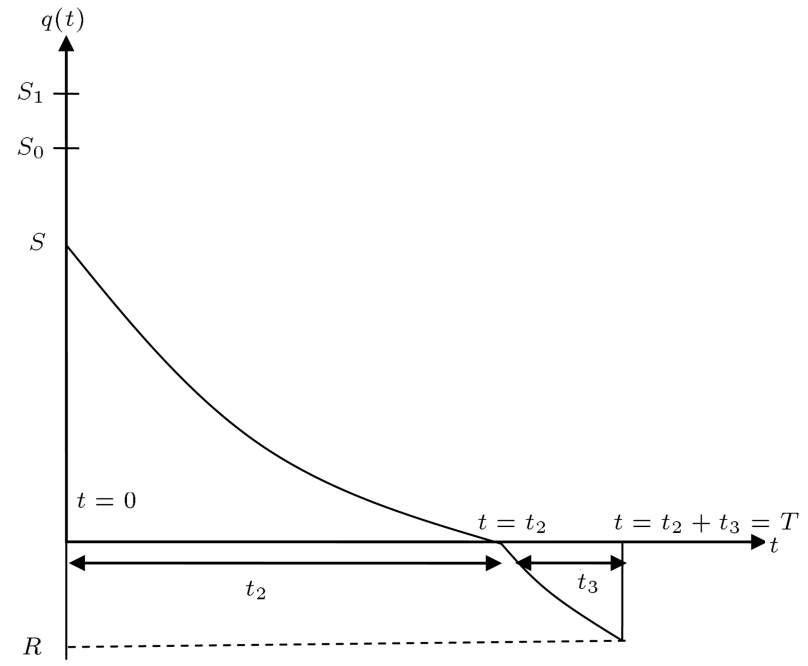

Figure 3. Inventory behavior of Scenario 3.

$$
\begin{aligned}
& \frac{d q(t)}{d t}+\theta q(t)=-f\left(A, p, S_{0}\right), \quad 0 \leq t \leq t_{2}, \\
& \frac{d q(t)}{d t}=-f\left(A, p, S_{0}\right) /[1+\delta(T-t)], \\
& t_{2}<t \leq t_{2}+t_{3}(=T)
\end{aligned}
$$

subject to the conditions that:

$$
\begin{aligned}
& q(t)=S \quad \text { at } \quad t=0, \\
& q(t)=0 \quad \text { at } \quad t=t_{2},
\end{aligned}
$$

and:

$$
q(t)=-R \quad \text { at } \quad t=t_{2}+t_{3}(=T) .
$$

The inventory behavior versus time for Scenario 3 is shown in Figure 3.

Using Conditions (19a)-(19c), the solutions to differential Eqs. (18a) and (18b) are as follows:

$$
\begin{aligned}
q(t) & =f\left(A, p, S_{0}\right)\left[\exp \left\{\theta\left(t_{2}-t\right)\right\}\right], \quad 0 \leq t \leq t_{2} \\
q(t) & =f\left(A, p, S_{0}\right) \log |1+\delta(T-t)| / \delta-R \\
t_{2} & <t \leq t_{2}+t_{3}(=T)
\end{aligned}
$$

Now, from Eqs. (19a) and (20a), it follows, therefore, that:

$$
S=f\left(A, p, S_{0}\right)\left\{\exp \left(\theta t_{2}\right)-1\right\} / \theta,
$$

which implies that:

$$
t_{2}=\log \left|\frac{f\left(A, p, S_{0}\right)+\theta S}{f\left(A, p, S_{0}\right)}\right| / \theta .
$$

Again, from Eqs. (19c) and (20b), it is clear that:

$$
R=f\left(A, p, S_{0}\right) \log \left|1+\delta t_{3}\right| / \delta,
$$

implying that:

$$
t_{3}=\exp \left(\frac{\delta R}{f\left(A, p, S_{0}\right)}-1\right) / \delta .
$$

During the stock-in period, the deteriorated units have to be separated. The total deteriorated units over the stock in period $\left(0 \leq t \leq t_{2}\right)$ are given by:

$$
\begin{aligned}
& D^{\prime}=S-\int_{0}^{t_{2}} D(A, p, q) d t, \\
& D^{\prime}=S-\int_{0}^{t_{2}} A^{\gamma}(a-b p+c q) d t, \\
& D^{\prime}=S-A^{\gamma}(a-b p)-A^{\gamma} c \int_{0}^{t_{2}} q(t) d t .
\end{aligned}
$$

The expression for $C_{\text {sho }}$ is the same as in Scenario 1 .

Hence, the net profit for this scenario during time period $T$ is given by:

$$
\begin{aligned}
X(A, S, R)= & \left(p-C_{3}\right)(S+R)-\left(\frac{C_{1}}{\theta}+p-p^{\prime}\right) D^{\prime} \\
& -C_{\text {sho }}-A G-C_{4}
\end{aligned}
$$

where $S$ and $R$ are continuous variables, and $A$ is an integer variable.

Therefore, for the fixed values of mark-up rates, $\lambda$ and $m$, profit function $Z_{3}$ (total profit per unit time for the entire cycle) of Scenario 3 of the system is given by:

$$
Z_{3}(A, S, R)=\frac{X}{T} .
$$

Hence, in this scenario, the optimization problem is as follows:

maximizing $Z_{3}(A, S, R)$,

subject to $S \leq W \leq S_{0}, R>0$, and $A$ is an integer.

\section{Solution procedure}

\subsection{Implementation of $G A$}

The optimal solution of the system can be obtained from the optimal solutions of each scenario. If (NP) denotes total profit per unit time of the inventory system, then $(\mathbf{N P})_{\text {opt }}=$ Maximize $Z_{j}(A, S, R), j=$ $1,2,3$, and the corresponding values of $A, S, R$ will be, respectively, the optimal solutions.

Now, it is necessary to solve the optimization problem of each scenario by a suitable method. Herein, the optimization problem of each scenario is a mixed 
integer nonlinear programming problem. For solving these problems, a Real Coded Genetic Algorithm (RCGA) is developed for integer as well as continuous variables.

The working principle of the developed RCGA is given in the following algorithm:

Step 1: Initialize the parameters of RCGA, bounds of the decision variables, and the values of the system parameters of the optimization problem of each scenario;

Step 2: $\tau \leftarrow 0$ ( $\tau$ presents the number of current generation/iteration);

Step 3: Initialize the chromosomes of population $P(\tau) \quad(P(\tau)$ represents the population at the $\tau$ th generation/iteration);

Step 4: Evaluate the fitness function for each chromosome of $P(\tau)$;

Step 5: Find the best chromosome from $P(\tau)$ according to the fitness value of each chromosome;

Step 6: Repeat the following until the termination criterion is satisfied:

(i) $\tau$ is increased by one $(\tau \leftarrow \tau+1)$;

(ii) Create a new population from the population of earlier generation, i.e. from $P(\tau-1)$ by exponential rank-based selection;

(iii) Improve the current population by crossover and mutation operations;

(iv) Evaluate the fitness of each chromosome of $P(\tau)$;

(v) Find the best chromosome according to the fitness value of each chromosome;

(vi) Compare the best chromosomes of current population, $P(\tau)$, and population, $P(\tau-1)$, of previous generation and store the better one.

Step 7: Print the result of the best chromosome/solution;

\section{Step 8: End.}

There are different parameters used in the genetic algorithm, viz. population size (p_size), maximum number of generation $\left(m_{-} g e n\right)$, probability of crossover ( $\left.p \_c r o s s\right)$, and probability of mutation ( $\left.p \_m u t e\right)$. Regarding the choice of population size, there is no hard and fast rule. However, if the population size is considered to be large, data storage in the intermediate steps of GA may create some problems at the computation time with the help of computer. On the other hand, for very small population size, some genetic operations cannot be implemented. Particularly, mutation operator does not work properly as the mutation rate is very low. Regarding the maximum number of generations, there is no clear indication of this value being considered at all. It depends upon the number of variables and the size of the search space of the optimization problem. Further, based on the natural genetics, it is well known that the probability of crossover is always greater than that of mutation. Usually, the probability of crossover oscillates between 0.60 to 0.95 , whereas that of mutation oscillates between 0.10 to 0.15 .

For proper and successful functioning of GA, the design of an appropriate chromosome of solutions of the problem is an important task. This work uses real numbers to represent the chromosomes in GA. In this case, a chromosome is coded in the form of vector of integer and/or floating point numbers, and every component of that chromosome represents a decision variable of the problem.

The selection process is one of the most important factors in the genetic algorithm. It is dependent on the well-known evolutionary principle "survival of the fittest". The primary objective of the operator is to emphasize the above average solutions and eliminate the below average solutions from the population for the next generation. This is achieved by performing the following tasks:

(i) Identify good (usually above average) chromosomes/solutions in a population;

(ii) Make multiple copies of good chromosomes/ solutions;

(iii) Eliminate bad chromosomes/solutions from the population so that multiple copies of good solutions can be placed in the population.

This work uses exponential ranking selection [47] as a selection operator. After the selection process, the resulting chromosomes (those which have survived) undergo genetic operations such as crossover and mutation. Crossover is an operation that really empowers the GA. It operates on two or more parent solutions at a time and generates offspring by recombining the features of the both parent solutions. In the optimization problem, there are two types of decision variables, e.g. integer and continuous variables. To fulfill the purpose, this work uses whole arithmetical crossover [47] for continuous variables and intermediate crossover [50] for integer variables.

For mutation operation, this work uses the nonuniform mutation for continuous variables, whereas uniform mutation is used for integer variables. It is noted that the action of non-uniform mutation [47] is dependent on the age of the population.

\subsection{Implementation of $P S O$}

A number of researchers have successfully used metaheuristic methods to solve complicated optimization problems in different fields of scientific and engineering disciplines. Some of these algorithms are simulated annealing, tabu search, genetic algorithm, particle 
swarm optimization, ant colony optimization, among others. Among these algorithms, Particle Swarm Optimization (PSO), as a widely used algorithm, has been employed for solving the optimization problems mentioned earlier.

Particle Swarm Optimization (PSO) is a population-based heuristic global search algorithm based on the social interaction and individual experience. It was proposed by Eberhart and Kennedy [51], Kennedy and Eberhart [52]. It has been widely used in finding the solutions to optimization problems. This algorithm is inspired by social behavior of bird flocking or fish schooling. In PSO, the potential solutions, called particles, fly through the search space of the problem by following the current optimum particles. PSO is initialized with a population of random particles positions (solutions), and then searches for an optimum solution in generation to generation. In every iteration, each particle is updated with two best positions (solutions). The first one is the best position (solution) reached so far by the particle, and this best position is said to be the personal best position, called $p_{i}^{(k)}$. The other one is the current best position (solution), obtained so far by any particle in the population. This best value is a global best, called $p_{g}^{(k)}$.

In each generation, the velocity and position of the $i$ th $\left(i=1,2, \cdots, p_{\_}\right.$size $)$particle are updated by the following rules:

$$
\begin{aligned}
v_{i}^{(k+1)}= & w v_{i}^{(k)}+c_{1} r_{1}\left(p_{i}^{(k)}-x_{i}^{(k)}\right) \\
& +c_{2} r_{2}\left(p_{g}^{(k)}-x_{i}^{(k)}\right)
\end{aligned}
$$

and:

$$
x_{i}^{(k+1)}=x_{i}^{(k)}+v_{i}^{(k+1)},
$$

where $w$ is the inertia weight, and $k(=1,2, \cdots, m-$ gen) indicates the iterations (generations). Constants $c_{1}(>0)$ and $c_{2}(>0)$ are the cognitive learning and social learning rates, respectively, which are the acceleration constants responsible for varying the particle velocity towards $p_{i}^{(k)}$ and $p_{g}^{(k)}$, respectively.

From Eq. (26), it is clear that the updated velocity of the $i$ th particle is calculated by considering three components:

(i) Previous velocity of the particle;

(ii) The distance between the particle's best previous and current positions;

(iii) The distance between swarm's best experience (the position of the best particle in the swarm) and the current position of the particle.

The velocity in Eq. (26) is also limited by the range $\left[-v_{\max }, v_{\max }\right]$ where $v_{\max }$ is called the maximum velocity of the particle. The choice of a too small value for $v_{\max }$ can cause very small updating of velocities and positions of particles at each iteration. Hence, the algorithm may take a long time to converge and face the problem of getting stuck to local minima. To overcome these situations, Clerc [53], Clerc and Kennedy [54] proposed improved velocity update rules employing constriction factor, $\chi$. According to them, the updated velocity is given by:

$$
\begin{aligned}
v_{i}^{(k+1)}= & \chi\left[v_{i}^{(k)}+c_{1} r_{1}\left(p_{i}^{(k)}-x_{i}^{(k)}\right)\right. \\
& \left.+c_{2} r_{2}\left(p_{g}^{(k)}-x_{i}^{(k)}\right)\right] .
\end{aligned}
$$

Herein, constriction factor, $\chi$, is expressed as follows:

$$
\chi=\frac{2}{\left|2-\phi-\sqrt{\phi^{2}-4 \phi}\right|},
$$

where $\phi=c_{1}+c_{2}, \phi>4$, and $\chi$ is a function of $c_{1}$ and $c_{2}$. Typically, $c_{1}$ and $c_{2}$ are both set to be 2.05 . Thus, $\phi$, is set to 4.1 and constriction coefficient, $\chi$, is 0.729. This PSO is known as PSO-CO, i.e. constriction coefficient-based PSO.

The search procedure of the particle swarm optimization can be summarized as follows:

Step 1: Initialize the PSO parameters and bounds of the decision variables of the optimization problem;

Step 2: Initialize a population of particles with random positions and velocities;

Step 3: Evaluate the fitness of all particles;

Step 4: Keep track of the locations where each individual has its highest fitness so far;

Step 5: Keep track of the position with the global best fitness;

Step 6: Update the velocity of each particle;

Step 7: Update the position of each particle;

Step 8: If the stopping criterion is satisfied, go to Step 9, otherwise go to Step 3;

Step 9: Print the position and fitness of global best particle;

\section{Step 10: End.}

\section{Numerical illustration}

To illustrate the proposed model, a numerical example with the following data has been considered. $C_{1}=$ Rs.3.0/unit/unit time, $C_{2}=$ Rs.10.0/unit/unit time, $C_{3}=$ Rs.15.0/unit/unit time, $m=0.80, C_{4}=$ Rs.150.0/unit/unit time, $a=200, b=0.5, c=0.3$, $\gamma=0.3, \delta=2.5, W=300$ units, $\theta=0.08, S_{1}=150.0$, $S_{0}=50.0, G=$ Rs.50.0/advertisement. 
Table 1. Best found solutions for different values of $\lambda$ by GA.

\begin{tabular}{cccccccccc}
\hline $\boldsymbol{\lambda}$ & $\boldsymbol{A}$ & $\boldsymbol{Z}$ & $\boldsymbol{S}$ & $\boldsymbol{R}$ & $\boldsymbol{t}_{\mathbf{0}}$ & $\boldsymbol{t}_{\mathbf{1}}$ & $\boldsymbol{t}_{\boldsymbol{2}}$ & $\boldsymbol{t}_{\boldsymbol{3}}$ & $\boldsymbol{T}$ \\
\hline 1.30 & 3 & 519.0371 & 294.0956 & 43.26 & 0.4179 & 0.3189 & 0.1740 & 0.1843 & 1.0951 \\
1.35 & 5 & 761.2683 & 381.8383 & 51.1629 & 0.5769 & 0.2750 & 0.1497 & 0.1880 & 1.1896 \\
1.40 & 6 & 1030.5967 & 425.2664 & 51.0656 & 0.6487 & 0.2611 & 0.1420 & 0.1760 & 1.2279 \\
1.45 & 7 & 1320.9511 & 466.7507 & 50.5739 & 0.7129 & 0.2500 & 0.1359 & 0.1651 & 1.2639 \\
1.50 & 8 & 1630.1554 & 506.5724 & 49.8235 & 0.7713 & 0.2407 & 0.1308 & 0.1551 & 1.2979 \\
\hline
\end{tabular}

Table 2. Best found solutions for different values of $\lambda$ by PSO.

\begin{tabular}{cccccccccc}
\hline $\boldsymbol{\lambda}$ & $\boldsymbol{A}$ & $\boldsymbol{Z}$ & $\boldsymbol{S}$ & $\boldsymbol{R}$ & $\boldsymbol{t}_{\mathbf{0}}$ & $\boldsymbol{t}_{\boldsymbol{1}}$ & $\boldsymbol{t}_{\boldsymbol{2}}$ & $\boldsymbol{t}_{\boldsymbol{3}}$ & $\boldsymbol{T}$ \\
\hline 1.30 & 3 & 519.0372 & 294.0966 & 43.27 & 0.418 & 0.3189 & 0.1741 & 0.1842 & 1.0952 \\
1.35 & 5 & 761.2684 & 381.8384 & 51.1630 & 0.5775 & 0.2765 & 0.1497 & 0.1885 & 1.1895 \\
1.40 & 6 & 1030.5988 & 425.2675 & 51.0675 & 0.6475 & 0.2610 & 0.1415 & 0.1775 & 1.2280 \\
1.45 & 7 & 1320.9535 & 466.7511 & 50.5745 & 0.7125 & 0.2502 & 0.1361 & 0.1652 & 1.2641 \\
1.50 & 8 & 1630.1585 & 506.5731 & 49.8242 & 0.7715 & 0.2409 & 0.1309 & 0.1555 & 1.2975 \\
\hline
\end{tabular}

The values of the above parameters considered in this numerical example are not selected from real-life case study; however, these values considered here are realistic. To obtain the solution of this highly nonlinear optimization problem, two soft-computing techniques, i.e. genetic algorithm and particle swarm optimization, are used. These algorithms are coded in c programming language and the computational runs have been done by a PC with Intel Core-2-due $2.5 \mathrm{GHz}$ Processor in LINUX environment.

This example is solved in order to find the values of $A, S, R$ and $t_{0}, t_{1}, t_{2}, t_{3}, T$ along with the total profit per unit time $(Z)$ of the inventory system. For this purpose, 20 independent runs have been performed by the proposed GA, and a set of results has been obtained. From these results, the following result corresponding to the best total profit per unit time as the solution of the given example is shown in Tables 1 and 2 .

In the proposed GA, the following values of GA parameters have been used:

$$
\begin{array}{ll}
\text { p_size }=50, & m \_g e n=200, \\
\text { p_cross }=0.9, & \text { p_mute }=0.1 .
\end{array}
$$

In the proposed PSO, the following values of PSO parameters have been used:

$$
\begin{array}{ll}
p \_ \text {size }=100, & m_{-} \text {gen }=100, \\
c_{1}=2.05, & c_{2}=2.05 .
\end{array}
$$

\section{Sensitivity analysis}

For the given numerical example mentioned in Section 5, sensitivity analyses have been performed to study the effect of changes of different parameters on initial stock level $(S)$, shortage level $(R)$, frequency of advertisement $(A)$, and cycle length $(T)$ along with the maximum profit of the inventory system $(Z)$. These analyses have been carried out by changing (increasing and decreasing) the parameters from $-20 \%$ to $+20 \%$. The results are obtained by changing one parameter at a time and keeping the other parameters at their original values. In each case of this analysis, 20 independent runs have been performed by the proposed GA, and a set of results has been considered. Among these results, the best found results have been considered. These results are shown in Table 3 , which are self-explanatory.

\subsection{Research objective and managerial insight}

The main objective of the proposed inventory model is to formulate a real-life business problem mathematically and solve the problem. This work describes a displayed stock-dependent demand and deteriorating inventory model. In real-life situations, the displayed stock plays an important role of demand function and attracts customers to buy more products. This type of problem is a highly nonlinear problem. For this reason, it is not possible to obtain the closed form in order to optimize the inventory problem. So, this type of problem is solved by two soft-computing algorithms (genetic algorithm and particle swarm optimization).

\section{Concluding remarks}

In reality, there are so many physical goods which deteriorate due to different factors such as dryness, damageability, perishability, etc. Therefore, these natural phenomena play an important role in any decision- 
Table 3. Sensitivity analysis with respect to different parameters.

\begin{tabular}{|c|c|c|c|c|c|c|}
\hline \multirow{2}{*}{ Parameters } & \multirow{2}{*}{$\%$ Changes } & \multirow{2}{*}{$\%$ Changes in $Z^{*}$} & \multirow{2}{*}{$A^{*}$} & \multicolumn{3}{|c|}{$\%$ Changes in } \\
\hline & & & & $S^{*}$ & $\boldsymbol{R}^{*}$ & $T^{*}$ \\
\hline \multirow{4}{*}{$C_{1}$} & -20 & 11.94 & 8 & 31.41 & 5.63 & 15.23 \\
\hline & -10 & 5.61 & 7 & 14.97 & 3.71 & 7.25 \\
\hline & 10 & -4.92 & 6 & -4.88 & 5.79 & -3.07 \\
\hline & 20 & -9.52 & 6 & -9.40 & 11.30 & -5.70 \\
\hline \multirow{4}{*}{$C_{2}$} & -20 & 0.73 & 6 & -0.32 & 9.74 & 1.25 \\
\hline & -10 & 0.35 & 6 & -0.03 & 4.65 & 0.59 \\
\hline & 10 & -0.32 & 6 & 0.48 & -4.25 & -0.53 \\
\hline & 20 & -0.61 & 6 & 0.69 & -8.16 & -1.01 \\
\hline \multirow{4}{*}{$C_{3}$} & -20 & -39.15 & 4 & -19.20 & -6.16 & -6.65 \\
\hline & -10 & -20.24 & 5 & -9.16 & -2.43 & -3.19 \\
\hline & 10 & 21.62 & 8 & 17.11 & 10.86 & 6.25 \\
\hline & 20 & 43.90 & 8 & 17.53 & 2.27 & 5.69 \\
\hline \multirow{4}{*}{$C_{4}$} & -20 & 2.39 & 6 & -1.56 & -2.77 & -1.90 \\
\hline & -10 & 1.19 & 6 & -0.66 & -1.38 & -0.94 \\
\hline & 10 & -1.18 & 6 & 1.12 & 1.38 & 0.94 \\
\hline & 20 & -2.37 & 7 & 10.31 & 11.15 & 5.29 \\
\hline \multirow{4}{*}{$a$} & -20 & -28.17 & 5 & -16.27 & -18.75 & 7.83 \\
\hline & -10 & -14.37 & 6 & -3.93 & -4.39 & 5.41 \\
\hline & 10 & 14.96 & 7 & 12.97 & 15.00 & -1.23 \\
\hline & 20 & 30.25 & 7 & 17.15 & 19.42 & -5.39 \\
\hline \multirow{4}{*}{$b$} & -20 & 1.52 & 6 & 0.66 & 0.45 & -0.52 \\
\hline & -10 & 0.76 & 6 & 0.46 & 0.23 & -0.25 \\
\hline & 10 & -0.76 & 6 & 0.02 & -0.23 & 0.26 \\
\hline & 20 & -1.52 & 6 & -0.19 & -0.45 & 0.53 \\
\hline \multirow{4}{*}{$c$} & -20 & -5.13 & 6 & -2.65 & 2.82 & 1.11 \\
\hline & -10 & -2.57 & 6 & -1.20 & 1.41 & 0.55 \\
\hline & 10 & 2.58 & 6 & 1.66 & -1.41 & -0.55 \\
\hline & 20 & 5.18 & 6 & 3.08 & -2.82 & -1.10 \\
\hline \multirow{4}{*}{$S_{0}$} & -20 & -0.47 & 6 & 0.59 & -2.62 & 0.24 \\
\hline & -10 & -0.24 & 6 & 0.42 & -1.31 & 0.12 \\
\hline & 10 & 0.25 & 6 & 0.05 & 1.30 & -0.14 \\
\hline & 20 & 0.51 & 6 & -0.15 & 2.58 & -0.30 \\
\hline \multirow{4}{*}{$S_{1}$} & -20 & -4.04 & 6 & -3.46 & 4.74 & -0.12 \\
\hline & -10 & -1.98 & 6 & -1.64 & 2.32 & -0.11 \\
\hline & 10 & 1.91 & 6 & 2.16 & -2.21 & 0.21 \\
\hline & 20 & 3.94 & 6 & 4.15 & -4.33 & 0.51 \\
\hline
\end{tabular}


Table 3. Sensitivity analysis with respect to different parameters (continued).

\begin{tabular}{|c|c|c|c|c|c|c|}
\hline \multirow{2}{*}{ Parameters } & \multirow{2}{*}{$\%$ Changes } & \multirow{2}{*}{$\%$ Changes in $Z^{*}$} & \multirow{2}{*}{$A^{*}$} & \multicolumn{3}{|c|}{$\%$ Changes in } \\
\hline & & & & $S^{*}$ & $\boldsymbol{R}^{*}$ & $T^{*}$ \\
\hline \multirow{4}{*}{$\theta$} & -20 & 1.12 & 6 & 0.89 & -1.30 & 0.98 \\
\hline & -10 & 0.56 & 6 & 0.56 & -0.65 & 0.48 \\
\hline & 10 & -0.56 & 6 & -0.09 & 0.65 & -0.48 \\
\hline & 20 & -1.11 & 6 & -0.41 & 1.29 & -0.94 \\
\hline \multirow{4}{*}{$\delta$} & -20 & 0.70 & 6 & -0.29 & 10.66 & 0.77 \\
\hline & -10 & 0.34 & 6 & -0.02 & 5.05 & 0.37 \\
\hline & 10 & -0.31 & 6 & 0.47 & -4.57 & -0.34 \\
\hline & 20 & -0.59 & 6 & 0.67 & -8.75 & -0.67 \\
\hline \multirow{4}{*}{$\gamma$} & -20 & -14.37 & 4 & -21.44 & -23.30 & -3.40 \\
\hline & -10 & -8.61 & 4 & -19.69 & -22.44 & -5.50 \\
\hline & 10 & 10.42 & 8 & 20.51 & 22.62 & 3.47 \\
\hline & 20 & 22.51 & 8 & 24.54 & 24.66 & 0.11 \\
\hline \multirow{4}{*}{$G$} & -20 & 11.57 & 8 & 7.36 & 6.06 & -2.08 \\
\hline & -10 & 5.26 & 8 & 12.09 & 13.41 & 2.51 \\
\hline & 10 & -9.89 & 6 & 3.72 & 5.47 & 3.71 \\
\hline & 20 & -8.63 & 5 & -2.63 & -1.45 & 2.93 \\
\hline
\end{tabular}

making process of any inventory system. When the deterioration effect of a good is high, the problem of finding a trade-off among holding, deterioration, and backordering costs is a complex problem. To avoid complexity in the formulation, many researchers have used Taylor's series approximation for exponential function. This work solved the problem without considering the Taylor's series approximation. Also, the results by PSO and GA were compared.

In the present model, the demand rate is considered as follows:

$$
\begin{aligned}
& D(A, p, q)=f\left(A, p, S_{1}\right), \quad q>S_{1}, \\
& D(A, p, q)=f(A, p, q), \quad S_{0} \leq q \leq S_{1}, \\
& D(A, p, q)=f\left(A, p, S_{0}\right), \quad q<S_{0},
\end{aligned}
$$

where:

$$
f(A, p, q)=A^{\gamma}(a-b p+c q) .
$$

Clearly, $D(A, p, q)$ is proportional to $(a-b p+c q)$ for some fixed $A$. On the other hand, for fixed $p$ and $q$, the demand of an item is likely to increase with the increase of the frequency of advertisement in the popular media. However, it is not directly proportional to the frequency of advertisement. Therefore, it is assumed that $D(A, p, q)$ is proportional to $A^{\gamma}(\gamma>0)$ for fixed $p$ and $q$.
This model is applicable to the differential items, foodgrains, fruits, green vegetables, among others. Additionally, this model can further be generalized to include the case of finite rate of replenishment of the stock as well as other possible functional relationships which may exist among the demand rate, selling price of the item, frequency of advertisement or the cost of advertisement, and displayed stock level in the showroom/shop.

\section{References}

1. Mandal, B.N. and Phaujdar, S. "An inventory model for deteriorating items and stock-dependent consumption rate", Journal of Operational Research Society, 40(5), pp. 483-488 (1989).

2. Pakkala, T.P.M. and Achary, K.K. "A deterministic inventory model for deteriorating items with two warehouse and finite replenishment rate", European Journal of Operational Research, 57(1), pp. 71-76 (1992).

3. Goyal, S.K. and Gunasekaran, A. "An integrated production-inventory-marketing model for deteriorating items", Computers \& Industrial Engineering, 28(4), pp. 755-762 (1995).

4. Giri, B.C., Pal, S., Goswami, A., and Chaudhuri, K.S. "An inventory model for deteriorating items with stock-dependent demand rate", European Journal of Operational Research, 95(3), pp. 604-610 (1996). 
5. Bhunia, A.K. and Maiti, M. "An inventory model for decaying items with selling price, frequency of advertisement and linearly time-dependent demand with shortages", IAPQR Transactions, 22, pp. 41-49 (1997).

6. Bhunia, A.K. and Maiti, M. "A two warehouses inventory model for deteriorating items with linear trend in demand and shortages", Journal of Operational Research Society, 49(3), pp. 287-292 (1997).

7. Luo, W. "An integrated inventory system for perishable goods with backordering", Computers \& Industrial Engineering, 34(3), pp. 685-693 (1998).

8. Chang, J. and Dye, C.Y. "An EOQ model for deteriorating items with time varying and partial backlogging", Journal of the Operational Research Society, 50(11), pp. 1176 -1182 (1999).

9. Abad, P.L. "Optimal lot size for a perishable good under conditions of finite production and partial backordering and lost sale", Computers \& Industrial Engineering, 38(4), pp. 457-468 (2000).

10. Pal, A.K., Bhunia, A.K., and Mukherjee, R.N. "Optimal lot size model for deteriorating items with demand rate dependent on displayed stock level (DSL) and partial backordering", European Journal of Operational Research, 175(2), pp. 977-991 (2006).

11. Dye, C.Y., Ouyang, L.Y., and Hsieh, T.P. "Deterministic inventory model for deteriorating items with the capability constraint and time-proportional backlogging rate", European Journal of Operational Research, 178(3), pp. 789-807 (2007).

12. Pal, P., Bhunia, A.K., and Goyal, S.K. "On optimal partially integrated production and marketing policy with variable demand under flexibility and reliability considerations via genetic algorithm", Applied Mathematics and Computation, 188(1), pp. 525-537 (2007).

13. Taleizadeh, A.A., Niaki, S.T., Shafii, N., Ghavamizadeh, Meibodi, R., and Jabbarzadeh, A. "A particle swarm optimization approach for constraint joint single buyer single vendor inventory problem with changeable lead-time and $(r, Q)$ policy in supply chain", International Journal of Advanced Manufacturing Technology, 51(9), pp. 1209-1223 (2010).

14. Bhunia, A.K., Pal, P., Chattopadhyay, S., and Medya, B.K. "An inventory model of two-warehouse system with variable demand dependent on instantaneous displayed stock and marketing decision via hybrid RCGA", International Journal of Industrial Engineering Computations, 2(2), pp. 351-368 (2011).

15. Bhunia, A.K. and Shaikh, A.A. "A two warehouse inventory model for deteriorating items with time dependent partial backlogging and variable demand dependent on marketing strategy and time", International Journal of Inventory Control and Management, 1, pp. 95-110 (2011).

16. Bhunia, A.K. and Shaikh, A.A. "A deterministic model for deteriorating items with displayed inventory level dependent demand rate incorporating marketing decisions with transportation cost", International Journal of Industrial Engineering Computations, 2, pp. 547562 (2011).

17. Liang, Y. and Zhou, F. "A two-warehouse inventory model for deteriorating items under conditionally permissible delay in payment", Applied Mathematical Modelling, 35(5), pp. 2221-2231 (2011).

18. Yang, H.L. "Two-warehouse partial backlogging inventory models with three-parameter Weibull distribution deterioration under inflation", International Journal of Production Economics, 138(1), pp. 107-116 (2012).

19. Taleizadeh, A.A., Pentico, D.W., Aryanezhad, M.B., and Ghoreyshi, M. "An economic order quantity model with partial backordering and a special sale price", European Journal of Operational Research, 221(3), pp. 571-583 (2012).

20. Taleizadeh, A.A., Niaki, S.T.A., and Makui, A. "Multi-product multi-chance constraint multi-buyer single-vendor supply chain problem with stochastic demand and variable lead time", Expert Systems with Applications, 39, pp. 5338-5348 (2012).

21. Bhunia, A.K., Shaikh, A.A., Maiti, A.K., and Maiti, M. "A two warehouse deterministic inventory model for deteriorating items with a linear trend in time dependent demand over finite time horizon by elitist real-coded genetic algorithm", International Journal Industrial Engineering and Computations, 4(2), pp. 241-258 (2013).

22. Yang, H.L. and Chang, C.T. "A two-warehouse partial backlogging inventory model for deteriorating items with permissible delay in payment under inflation", Applied Mathematical Modelling, 37(5), pp. 2717-2726 (2013).

23. Taleizadeh, A.A., Pentico, D.W., Jabalameli, M.S., and Aryanezhad, M.B. "An economic order quantity model with multiple partial prepayments and partial backordering", Mathematical and Computer Modelling, 57(3-4), pp. 311-323 (2013).

24. Taleizadeh, A.A., Pentico, D.W., Jabalameli, M.S. and Aryanezhad, M.B. "An EOQ problem under partial delayed payment and partial backordering", Omega, 41(2), pp. 354-368 (2013).

25. Taleizadeh, A.A., Wee, H.M., and Jolai, F. "Revisiting fuzzy rough economic order quantity model for deteriorating items considering quantity discount and prepayment", Mathematical and Computer Modeling, 57(5-6), pp. 1466-1479 (2013).

26. Bhunia, A.K. and Shaikh, A.A. "A deterministic inventory model for deteriorating items with selling price dependent demand and three-parameter Weibull distributed deterioration", International Journal of Industrial Engineering Computations, 5(3), pp. 497510 (2014).

27. Bhunia, A.K., Mahato, S.K., Shaikh, A.A., and Jaggi, C.K. "A deteriorating inventory model with displayed stock-level-dependent demand and partially 
backlogged shortages with all unit discount facilities via particle swarm optimization", International Journal of System Science Operations and Logistic, 1(3), pp. 164-180 (2015).

28. Bhunia, A.K., Shaikh, A.A., and Gupta, R.K. "A study on two-warehouse partially backlogged deteriorating inventory models under inflation via particle swarm optimization", International Journal of System Science, 46(6), pp. 1036-1050 (2015).

29. Bhunia, A.K., Shaikh, A.A., Sharma, G., and Pareek, S. "A two storage inventory model for deteriorating items with variable demand and partial backlogging", Journal of Industrial and Production Engineering, 32(4), pp. 263-272 (2015).

30. Mondal, B., Bhunia, A.K., and Maiti, M. "A model of two storage inventory system under stock dependent selling rate incorporating marketing decisions and transportation cost with optimum release rule", Tamsui Oxford Journal of Mathematical Sciences, 23(3), pp. 243-267 (2007).

31. Baker, R.C. and Urban, T.L. "A deterministic inventory system with an inventory-level-dependent demand rate", Journal of Operational Research Society, 39(9), pp. 1823-1831 (1988).

32. Datta, T.K., and Pal, A.K. "A note on an inventory model with inventory-level-dependent demand rate", Journal of the Operational Research Society, 41(10), pp. $971-975$ (1990).

33. Urban, T.L. "An inventory model with an inventorylevel-dependent rate and relaxed terminal conditions", Journal of Operational Research Society, 43(7), pp. 721-724 (1992).

34. Paul, K., Datta, T.K., Chaudhuri, K.S., and Pal, A.K. "An inventory model with two component demand rate and shortages", Journal of the Operational Research Society, 47(8), pp. 1029-1036 (1996).

35. Urban, T.L. "Deterministic inventory models incorporating marketing decisions", Computers \& Industrial Engineering, 22(1), pp. 85-93 (1992).

36. Abad, P.L. "Optimal pricing and lot-sizing when the supplier offers a temporary price reduction over an interval", Computers \& Industrial Engineering, 30(1), pp. 63-74 (2003).

37. Pal, A.K., Bhunia, A.K. and Mukherjee, R.N. "A marketing-oriented inventory model with threecomponent demand rate dependent on displayed stock level (DSL)", Journal of the Operational Research Society, 56, pp. 113-118 (2004).

38. Sarkar, B. "An EOQ model with delay-in-payments and time-varying deterioration rate", Mathematical and Computer Modelling, 55(3-4), pp. 367-377 (2012).

39. Sarkar, B. "A production-inventory model with probabilistic deterioration in two-echelon supply chain management", Applied Mathematical Modelling, 37(5), pp. 3138-3151 (2013).
40. Sarkar, B. "Supply chain coordination with variable backorder, inspections, and discount policy for fixed lifetime products", Mathematical Problem in Engineering, Article ID 6318737, 14 pages (2016).

41. Sarkar, B., Saren, S., and Cárdenas-Barrón, L.E. "An inventory model with trade-credit policy and variable deterioration for fixed lifetime products", Annals of Operations Research, 229(1), pp. 677-702 (2015).

42. Sarkar, B., Mandal, P., and Sarkar, S. "An EMQ model with price and time dependent demand under the effect of reliability and inflation", Applied Mathematics and Computation, 231, pp. 414-421 (2014).

43. Sarkar, B. and Sarkar, S. "An improved inventory model with partial backlogging, time varying deterioration and stock-dependent demand", Economic Modelling, 30, pp. 924-932 (2013).

44. Sarkar, B. and Sarkar, S. "Variable deterioration and demand-An inventory model", Economic Modelling, 31, pp. 548-556 (2013).

45. Goldberg, D.E., Genetic Algorithms: Search, Optimization and Machine Learning, Addison Wesley, Reading, MA (1989).

46. Holland, J.H., Adaptation of Natural and Artificial System, University of Michigan Press, Ann Arbor (1975).

47. Michalawicz, Z., Genetic Algorithms + Data structure = Evolution Programs, Springer-Verlag, Berlin (1992).

48. Deb, K., Optimization for Engineering Design Algorithms and Examples, Prentice-Hall of India, New Delhi (1995).

49. Sakawa, M., Genetic Algorithms and Fuzzy MultiObjective Optimization, Kluwer Academic Publishers (2002).

50. Bhunia, A.K., Sahoo, L., and Roy, D. "Reliability stochastic optimization for a series system with interval component reliability via genetic algorithm", Applied Mathematics and Computation, 216(3), pp. 929-939 (2010).

51. Eberhart, R.C. and Kennedy, J.F. "A new optimizer using particle swarm theory", In Proceeding of the Sixth International Symposium on Micro Machine and Human Science, Nagoya, Japan, pp. 39-43 (1995).

52. Kennedy, J.F. and Eberhart, R.C. "Particle swarm optimization", In Proceeding of the IEEE International Conference on Neural Network, IV, Perth, Australia, pp. 1942-1948 (1995).

53. Clerc, M. "The swarm and queen: towards a deterministic and adaptive particle swarm optimization", In Proceedings of IEEE Congress on Evolutionary Computation, Washington, DC, USA, pp. 1951-1957 (1999).

54. Clerc, M. and Kennedy, J.F. "The particle swarm: explosion, stability, and Convergence in a multidimensional complex space", IEEE Trans Evol Comput., 6(1), pp. 58-73 (2002). 


\section{Biographies}

Asoke Kumar Bhunia is a Professor at the Department of Mathematics, The University of Burdwan, India. His research interests include computational optimization, soft computing, interval mathematics, interval ranking, etc. He has published more than one hundred research papers in various international SCI journals. He is a reviewer of several Elsevier Science, Springer, Taylor \& Francis, and other SCI journals. He has guided nine $\mathrm{PhD}$ and two MPhil students. He has also written three book chapters, two research monographs and a book entitled Advanced Operations Research. He is an INSA Visiting Fellow and also an Associate Editor of the Springer journal OPSEARCH.

Ali Akbar Shaikh is currently working as a Postdoctoral Fellow at School of Engineering and Sciences, Tecnológico de Monterrey, E. Garza Sada 2501 Sur, C.P. 64849, Monterrey, Nuevo León, México. He obtained MSc degree in Applied Mathematics from University of Kalyani, India, MPhil and $\mathrm{PhD}$ in Mathematics from University of Burdwan, India. Dr. Shaikh has published 14 research papers in different peer-reviewed and JCR international journals. His research interests include inventory control theory, interval optimization, and particle swarm optimization.

Vinti Dhaka is a Research Associate at the Department of Mathematics and Statistics, Banasthali Vidyapith, India. She completed her MPhil in Mathematical Sciences in 2012 and PhD in Opera- tional Research in 2016 from Banasthali Vidyapith. She has published four research papers in international/national journals and one chapter in book. Her area of interest is Inventory Management.

Sarla Pareek is a Professor at the Department of Mathematics and Statistics, Banasthali Vidyapith, India. She is a life member of various academic bodies. She has published 3 Book Chapters and more than 50 papers in various international/national journals including IJIEC, ADS, AMM, IJLSM, IJSDS etc. She is a reviewer in various international/national journals. Her area of research includes inventory management, demography and applied statistics. She has guided 9 MPhil and 15 PhD students.

Leopoldo Eduardo Cárdenas-Barrón is currently a Professor at School of Engineering and Sciences at Tecnológico de Monterrey, Campus Monterrey, México. $\mathrm{He}$ is also a Faculty Member at the Department of Industrial and Systems Engineering at Tecnológico de Monterrey. He was the Associate Director of the Industrial and Systems Engineering programme from 1999 to 2005. Moreover, he was also the Associate Director of the Department of Industrial and Systems Engineering from 2005 to 2009. His research areas primarily include inventory planning and control, logistics, and supply chain. He has published papers and technical notes in international and national Journals. He has co-authored one book in the field of Simulation in Spanish. He is also an editorial board member in several international journals. 